Supplement of Atmos. Chem. Phys. Discuss., 15, 26089-26130, 2015

http://www.atmos-chem-phys-discuss.net/15/26089/2015/

doi:10.5194/acpd-15-26089-2015-supplement

(C) Author(s) 2015. CC Attribution 3.0 License.

(c) (i)

Supplement of

\title{
Multimodel emission metrics for regional emissions of short lived climate forcers
}

\section{B. Aamaas et al.}

Correspondence to: B. Aamaas (borgar.aamaas@cicero.oslo.no)

The copyright of individual parts of the supplement might differ from the CC-BY 3.0 licence. 
1 Not all details are given in the article. Exact emission metric values and additional figures are

2 provided below. There is no single emission metric and time horizon that fits all applications, and the

3 selection will depend on what aspects of climate change are considered to be most important

4 (Aamaas et al., 2013).

\section{1. Emission metric values and comparison with literature}

6 Emission metric values for GWP and GTP with time horizons 20 years and 100 years are given in

7 Table SI1. We provide also emission metric values for emissions on land outside of Europe and East

8 Asia. This category is labelled "rest of the world". Both values from our study and the literature are 9 given. 

included. The units used are given in the brackets, such as $\mathrm{NO}_{\mathrm{x}}$ are based on $\mathrm{N}$ basis.

\begin{tabular}{|c|c|c|c|c|}
\hline Species & GWP(20) & GWP(100) & GTP(20) & GTP(100) \\
\hline \multicolumn{5}{|l|}{$B C[C]$, this study } \\
\hline BC, Europe, summer & 2000 & 540 & 570 & 74 \\
\hline BC, Europe, winter & 1700 & 470 & 490 & 64 \\
\hline BC, East Asia, summer & 1300 & 370 & 390 & 50 \\
\hline BC, East Asia, winter & 1100 & 300 & 320 & 41 \\
\hline $\mathrm{BC}$, Rest of the world, summer & 2200 & 610 & 650 & 83 \\
\hline $\mathrm{BC}$, Rest of the world, winter & 24009 & 600 & 680 & 88 \\
\hline $\mathrm{BC}$, Shipping, NH summer & 1700 & 450 & 480 & 62 \\
\hline BC, Shipping, NH winter & 1800 & 480 & 510 & 66 \\
\hline BC, Global, NH summer & 2100 & 570 & 600 & 78 \\
\hline $\mathrm{BC}$, Global, NH winter & 2000 & 530 & 570 & 74 \\
\hline \multicolumn{5}{|l|}{ BC, other studies } \\
\hline $\begin{array}{l}\text { Bond et al. (2013), BC, total, global. Metric } \\
\text { values are given for total effect }\end{array}$ & 3200 & 900 & 920 & 130 \\
\hline Collins et al. (2013), BC (four regions) & 1200 & 345 & 420 & 56 \\
\hline Collins et al. (2013), BC, Europe & N/A & N/A & 530 & 71 \\
\hline Collins et al. (2013), BC, East Asia & N/A & N/A & 410 & 55 \\
\hline $\begin{array}{l}\text { Bond et al. (2011), BC, aerosol-radiation } \\
\text { interaction + albedo, global }\end{array}$ & 2900 & 830 & N/A & N/A \\
\hline Fuglestvedt et al. (2010), BC, global & 1600 & 460 & 470 & 64 \\
\hline \multicolumn{5}{|l|}{ OC [C], this study } \\
\hline OC, Europe, summer & -760 & -210 & -220 & -28 \\
\hline OC, Europe, winter & -390 & -110 & -110 & -15 \\
\hline OC, East Asia, summer & -480 & -130 & -140 & -18 \\
\hline OC, East Asia, winter & -180 & -49 & -52 & -6.8 \\
\hline OC, Rest of the world, summer & -710 & -190 & -210 & -27 \\
\hline OC, Rest of the world, winter & -720 & -190 & -210 & -27 \\
\hline OC, Shipping, NH summer & -2100 & -580 & -620 & -80 \\
\hline OC, Shipping, NH winter & -1100 & -290 & -310 & -40 \\
\hline OC, Global, NH summer & -680 & -180 & -200 & -25 \\
\hline OC, Global, NH winter & -570 & -150 & -160 & -21 \\
\hline \multicolumn{5}{|l|}{ OC, other studies } \\
\hline Bond et al. (2011), OC & -160 & -46 & N/A & N/A \\
\hline Collins et al. (2013), OC (four regions) & -160 & -46 & -55 & -7.3 \\
\hline Collins et al. (2013), OC, Europe & $\mathrm{N} / \mathrm{A}$ & N/A & -58 & -7.7 \\
\hline Collins et al. (2013), OC, East Asia & $\mathrm{N} / \mathrm{A}$ & N/A & -50 & -6.7 \\
\hline Fuglestvedt et al. (2010), OC, global & -240 & -69 & -71 & -10 \\
\hline
\end{tabular}




\begin{tabular}{|c|c|c|c|c|}
\hline Species & GWP(20) & GWP(100) & GTP(20) & GTP(100) \\
\hline \multicolumn{5}{|l|}{ SO2 [SO2], this study } \\
\hline SO2, Europe, summer & -430 & -120 & -130 & -16 \\
\hline SO2, Europe, winter & -140 & -38 & -41 & -5.3 \\
\hline SO2, East Asia, summer & -255 & -69 & -74 & -9.6 \\
\hline SO2, East Asia, winter & -104 & -28 & -30 & -3.9 \\
\hline SO2, Rest of the world, summer & -490 & -130 & -140 & -19 \\
\hline SO2, Rest of the world, winter & -320 & -90 & -92 & -12 \\
\hline SO2, Shipping, NH summer & -560 & -150 & -160 & -21 \\
\hline SO2, Shipping, NH winter & -390 & -110 & -110 & -15 \\
\hline SO2, Global, NH summer & -410 & -110 & -120 & -15 \\
\hline SO2, Global, NH winter & -230 & -60 & -70 & -8.5 \\
\hline \multicolumn{5}{|l|}{ SO2, other studies } \\
\hline Collins et al. (2013), SO2 (four regions) & N/A & N/A & -38 & -5.1 \\
\hline Collins et al. (2013), SO2, Europe & N/A & N/A & -43 & -5.7 \\
\hline Collins et al. (2013), SO2, East Asia & N/A & N/A & -31 & -4.1 \\
\hline Fuglestvedt et al. (2010), SO2, global & -140 & -40 & -41 & -5.7 \\
\hline \multicolumn{5}{|l|}{ NH3 [NH3], this study } \\
\hline NH3, Europe, summer & -55 & -15 & -16 & -2.0 \\
\hline $\mathrm{NH3}$, Europe, winter & -36 & -9.7 & -10 & -1.3 \\
\hline NH3, East Asia, summer & -27 & -7.2 & -7.7 & -1.0 \\
\hline NH3, East Asia, winter & -51 & -14 & -15 & -1.9 \\
\hline $\mathrm{NH} 3$, Rest of the world, NH summer & -21 & -5.7 & -6.0 & -0.78 \\
\hline $\mathrm{NH} 3$, Rest of the world, $\mathrm{NH}$ winter & -32 & -8.8 & -9.4 & -1.2 \\
\hline NH3, Global, NH summer & -25 & -6.7 & -7.2 & -0.9 \\
\hline $\mathrm{NH} 3$, Global, NH winter & -37 & -10 & -11 & -1.4 \\
\hline \multicolumn{5}{|l|}{ NH3, other studies } \\
\hline \multirow[t]{2}{*}{ NH3, Shindell et al. (2009) } & -80 & -23 & -23 & -3.2 \\
\hline & & & (continued & next page) \\
\hline
\end{tabular}




\begin{tabular}{|c|c|c|c|c|}
\hline Species & GWP(20) & GWP(100) & GTP(20) & GTP(100) \\
\hline \multicolumn{5}{|l|}{ NOx $[N]$, this study } \\
\hline NOx, Europe, summer & -110 & -36 & -90 & -5.1 \\
\hline NOx, Europe, winter & -61 & -19 & -40 & -2.7 \\
\hline NOx, East Asia, summer & -63 & -22 & -75 & -3.3 \\
\hline NOx, East Asia, winter & -140 & -41 & -75 & -5.8 \\
\hline NOx, Rest of the world, summer & -120 & -44 & -160 & -6.6 \\
\hline NOx, Rest of the world, winter & -180 & -61 & -190 & -8.9 \\
\hline NOx, Shipping, NH summer & 79 & -2.2 & -230 & -1.3 \\
\hline NOx, Shipping, NH winter & -250 & -98 & -400 & -15 \\
\hline NOx, Global, NH summer & -120 & -42 & -150 & -6.2 \\
\hline NOx, Global, NH winter & -170 & -55 & -160 & -8.1 \\
\hline \multicolumn{5}{|l|}{ NOx, other studies } \\
\hline $\begin{array}{l}\text { Collins et al. (2013), NOx (four regions), } \\
\text { based on Fry et al. (2012) with including } \\
\text { stratospheric H2O }\end{array}$ & -15.9 & -11.6 & -62.1 & -2.2 \\
\hline $\begin{array}{l}\text { Collins et al. (2013), NOx, Europe, based on } \\
\text { Fry et al. (2012) with including stratospheric } \\
\mathrm{H}_{2} \mathrm{O}\end{array}$ & -39.4 & -15.6 & -48.0 & -2.5 \\
\hline $\begin{array}{l}\text { Collins et al. (2013), NOx, East Asia, based } \\
\text { on Fry et al. (2012) with including } \\
\text { stratospheric } \mathrm{H}_{2} \mathrm{O}\end{array}$ & 6.4 & -5.3 & -55.6 & -1.3 \\
\hline $\begin{array}{l}\text { Shindell et al. (2009), NOx, global, including } \\
\text { direct and indirect aerosol effects }\end{array}$ & -560 & -159 & $\mathrm{~N} / \mathrm{A}$ & N/A \\
\hline \multicolumn{5}{|l|}{ Surface } \\
\hline $\begin{array}{l}\text { Naik et al. (2005), Tropics, as given by } \\
\text { Fuglestvedt et al. (2010) }\end{array}$ & 43 & -28 & -260 & -6.6 \\
\hline $\begin{array}{l}\text { Wild et al. (2001), Tropics, as given by } \\
\text { Fuglestvedt et al. (2010) }\end{array}$ & 130 & -9.7 & -220 & -5.4 \\
\hline $\begin{array}{l}\text { Naik et al. (2005), Mid-lat, as given by } \\
\text { Fuglestvedt et al. (2010) }\end{array}$ & -43 & -18 & -54 & -2.9 \\
\hline $\begin{array}{l}\text { Berntsen et al. (2005), UiO, Mid-lat, as given } \\
\text { by Fuglestvedt et al. (2010) }\end{array}$ & 23 & 1.6 & -37 & -0.024 \\
\hline $\begin{array}{l}\text { Berntsen et al. (2005), LMDz, Mid-lat, as } \\
\text { given by Fuglestvedt et al. (2010) }\end{array}$ & 23 & -6.3 & -55 & -2.4 \\
\hline $\begin{array}{l}\text { Wild et al. (2001), Mid-lat, as given by } \\
\text { Fuglestvedt et al. (2010) }\end{array}$ & -3.7 & -9.3 & -48 & -2.0 \\
\hline $\begin{array}{l}\text { Wild et al. (2001), Global, as given by } \\
\text { Fuglestvedt et al. (2010) }\end{array}$ & 19 & -11 & -87 & -2.9 \\
\hline \multicolumn{5}{|l|}{ Shipping } \\
\hline NOx, Shipping, Collins et al. (2010) & -107 & -73 & -135 & N/A \\
\hline $\begin{array}{l}\text { Eyring et al. (2007), as given by Fuglestvedt } \\
\text { et al. (2010) }\end{array}$ & -76 & -36 & -130 & -6.1 \\
\hline $\begin{array}{l}\text { Endresen et al. (2003), as given by } \\
\text { Fuglestvedt et al. (2010) }\end{array}$ & -47 & -32 & -190 & -5.3 \\
\hline $\begin{array}{l}\text { Fuglestvedt et al. (2008), as given by } \\
\text { Fuglestvedt et al. (2010) }\end{array}$ & -31 & -25 & -160 & -4.2 \\
\hline
\end{tabular}




\begin{tabular}{|c|c|c|c|c|}
\hline Species & GWP(20) & GWP(100) & GTP(20) & GTP(100) \\
\hline \multicolumn{5}{|l|}{ Aircraft } \\
\hline $\begin{array}{l}\text { Stevenson et al. (2004), as given by } \\
\text { Fuglestvedt et al. (2010) }\end{array}$ & 120 & -2.1 & -240 & -2.2 \\
\hline $\begin{array}{l}\text { Wild et al. (2001) (as in Stevenson et al. } \\
\text { (2004)), as given by Fuglestvedt et al. (2010) }\end{array}$ & 410 & 71 & -200 & 7.6 \\
\hline $\begin{array}{l}\text { Köhler et al. (2008), as given by Fuglestvedt } \\
\text { et al. (2010) }\end{array}$ & 470 & 6.9 & -590 & -9.5 \\
\hline \multicolumn{5}{|l|}{$\mathrm{CO}[\mathrm{CO}]$, this study } \\
\hline CO, Europe, summer & 7.3 & 2.2 & 4.3 & 0.31 \\
\hline CO, Europe, winter & 7.9 & 2.4 & 4.9 & 0.34 \\
\hline CO, East Asia, summer & 8.3 & 2.4 & 4.5 & 0.34 \\
\hline CO, East Asia, winter & 8.5 & 2.5 & 5.0 & 0.36 \\
\hline $\mathrm{CO}$, Rest of the world, summer & 7.5 & 2.2 & 4.4 & 0.31 \\
\hline $\mathrm{CO}$, Rest of the world, winter & 8.1 & 2.4 & 4.9 & 0.34 \\
\hline CO, Shipping, NH summer & 8.4 & 2.5 & 4.7 & 0.35 \\
\hline CO, Shipping, NH winter & 8.6 & 2.6 & 5.9 & 0.37 \\
\hline CO, Global, NH summer & 7.6 & 2.3 & 4.4 & 0.32 \\
\hline $\mathrm{CO}$, Global, NH winter & 8.2 & 2.4 & 4.9 & 0.35 \\
\hline \multicolumn{5}{|l|}{ CO, other studies } \\
\hline $\begin{array}{l}\text { Collins et al. (2013), } \mathrm{CO} \text { (four regions), based } \\
\text { on Fry et al. (2012) with including } \\
\text { stratospheric } \mathrm{H}_{2} \mathrm{O}\end{array}$ & 5.4 & 1.8 & 3.5 & 0.26 \\
\hline $\begin{array}{l}\text { Collins et al. (2013), CO, Europe, based on } \\
\text { Fry et al. (2012) with including stratospheric } \\
\mathrm{H}_{2} \mathrm{O}\end{array}$ & 4.9 & 1.6 & 3.2 & 0.24 \\
\hline $\begin{array}{l}\text { Collins et al. (2013), CO, East Asia, based on } \\
\text { Fry et al. (2012) with including stratospheric } \\
\mathrm{H}_{2} \mathrm{O}\end{array}$ & 5.4 & 1.8 & 3.5 & 0.26 \\
\hline $\begin{array}{l}\text { Shindell et al. (2009), CO, global, including } \\
\text { direct and indirect aerosol effects }\end{array}$ & 18.6 & 5.3 & N/A & N/A \\
\hline $\begin{array}{l}\text { CO, surface, Berntsen et al. (2005) UiO Mid- } \\
\text { lat, as given by Fuglestvedt et al. (2010) }\end{array}$ & 7.2 & 2.3 & 4.1 & 0.33 \\
\hline $\begin{array}{l}\text { CO, surface, Berntsen et al. (2005) LMDz } \\
\text { Mid-lat, as given by Fuglestvedt et al. (2010) }\end{array}$ & 9.3 & 3.3 & 6.1 & 0.55 \\
\hline $\begin{array}{l}\text { Derwent et al. (2001), as given by } \\
\text { Fuglestvedt et al. (2010), CO, surface }\end{array}$ & 6.0 & 2.0 & 3.7 & 0.27 \\
\hline
\end{tabular}




\begin{tabular}{|c|c|c|c|c|}
\hline Species & GWP(20) & GWP(100) & GTP(20) & GTP(100) \\
\hline \multicolumn{5}{|l|}{ VOC $[C]$, this study } \\
\hline VOC, Europe, summer & 41 & 12 & 23 & 1.7 \\
\hline VOC, Europe, winter & 27 & 7.9 & 14 & 1.1 \\
\hline VOC, East Asia, summer & 42 & 12 & 19 & 1.7 \\
\hline VOC, East Asia, winter & 11 & 3.6 & 8.9 & 0.51 \\
\hline VOC, Rest of the world, summer & 38 & 11 & 22 & 1.6 \\
\hline VOC, Rest of the world, winter & 40 & 12 & 25 & 1.7 \\
\hline VOC, Shipping, NH summer & 47 & 14 & 32 & 2.1 \\
\hline VOC, Shipping, NH winter & 45 & 14 & 30 & 1.9 \\
\hline VOC, Global, NH summer & 38 & 11 & 22 & 1.6 \\
\hline VOC, Global, NH winter & 35 & 10 & 21 & 1.5 \\
\hline \multicolumn{5}{|l|}{ VOC, other studies } \\
\hline $\begin{array}{l}\text { Collins et al. (2013), VOC (four regions), } \\
\text { based on Fry et al. (2012) with including } \\
\text { stratospheric } \mathrm{H}_{2} \mathrm{O}\end{array}$ & 18.7 & 5.8 & 10.0 & 0.9 \\
\hline $\begin{array}{l}\text { Collins et al. (2013), VOC, Europe, based on } \\
\text { Fry et al. (2012) with including stratospheric } \\
\mathrm{H}_{2} \mathrm{O}\end{array}$ & 18.0 & 5.6 & 9.5 & 0.8 \\
\hline $\begin{array}{l}\text { Collins et al. (2013), VOC, East Asia, based } \\
\text { on Fry et al. (2012) with including } \\
\text { stratospheric } \mathrm{H}_{2} \mathrm{O}\end{array}$ & 16.3 & 5.0 & 8.4 & 0.7 \\
\hline $\begin{array}{l}\text { Collins et al. (2002), as given by Fuglestvedt } \\
\text { et al. (2010), VOC, surface }\end{array}$ & 14 & 4.5 & 7.5 & 0.66 \\
\hline \multicolumn{5}{|l|}{ CH4 [CH4], this study } \\
\hline $\mathrm{CH} 4$, Global & 77 & 23 & 48 & 3.3 \\
\hline \multicolumn{5}{|l|}{ CH4, other studies } \\
\hline Myhre et al. (2013) & 84 & 28 & 67 & 4.3 \\
\hline
\end{tabular}



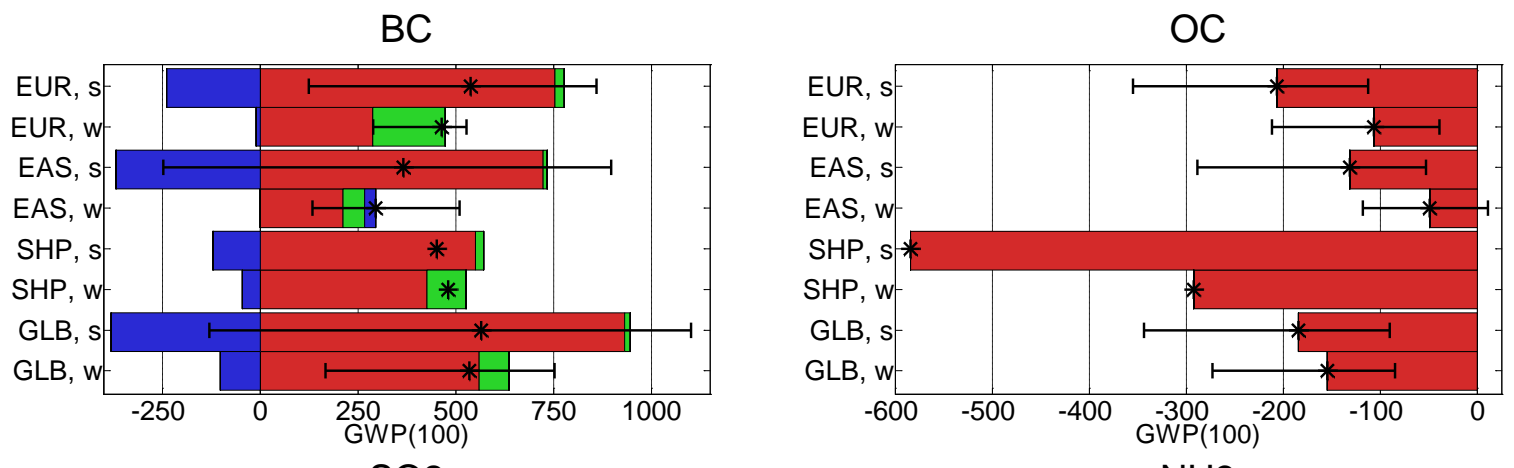

$\mathrm{SO} 2$
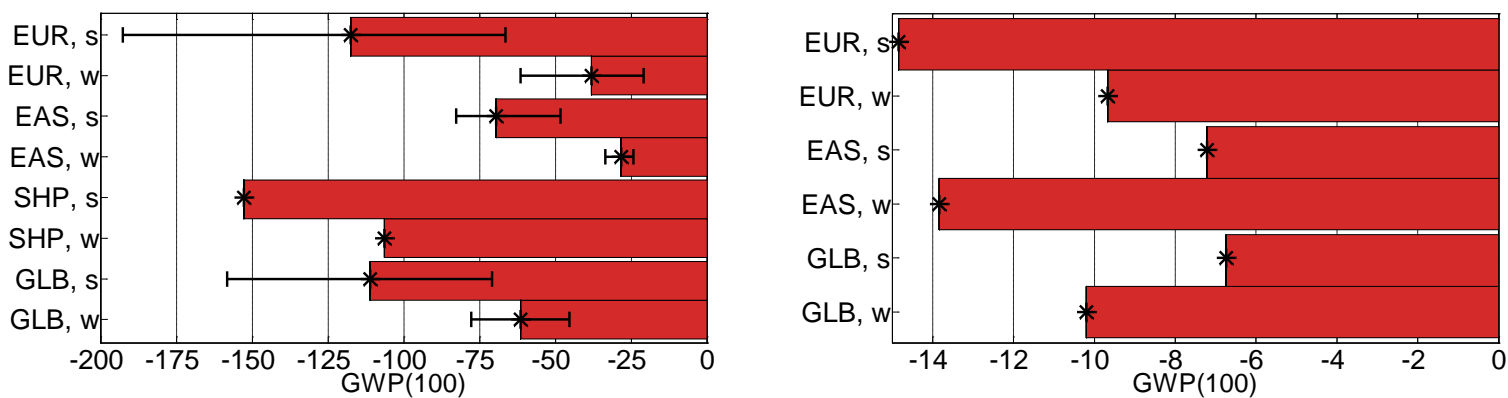

$\mathrm{CH} 4$

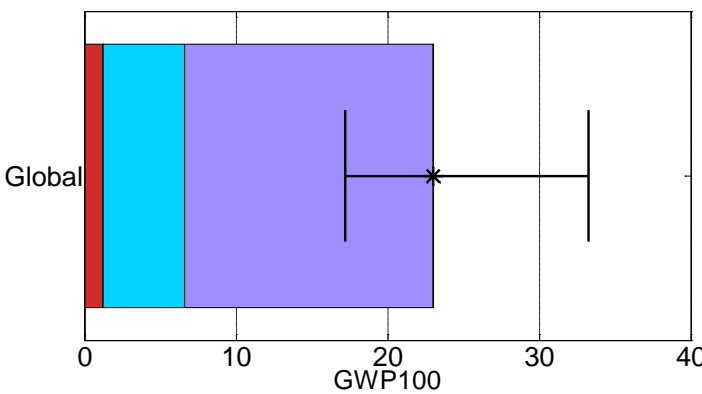

\section{aerosol effects \\ BC deposition on snow \\ BC semi-direct short-lived ozone methane methane-induced ozone *net effect (best estimate)}

Figure SI1: GWP(100) values for the species not shown in the article, for all regions and seasons, decomposed by processes. The regions included are Europe (EUR), East Asia (EAS), shipping (SHP), and global (GLB), all for both NH summer, May-October, (s) and NH winter, November-April, (w).

\section{Variations with time horizon}

Figure SI2 and GWP in Figure SI3. 
OC

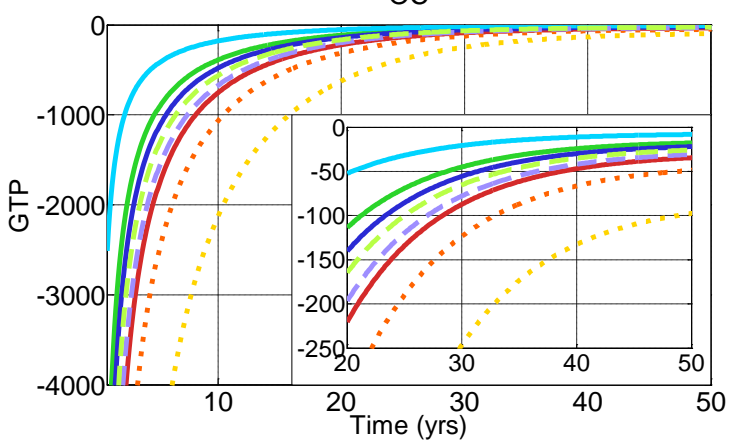

$\mathrm{NH} 3$

39

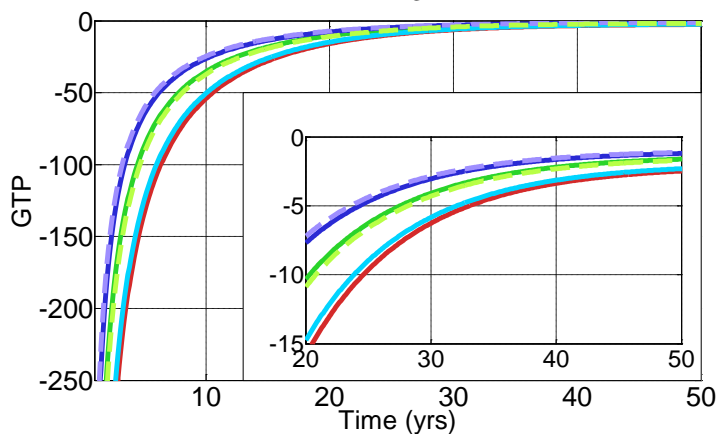

VOC

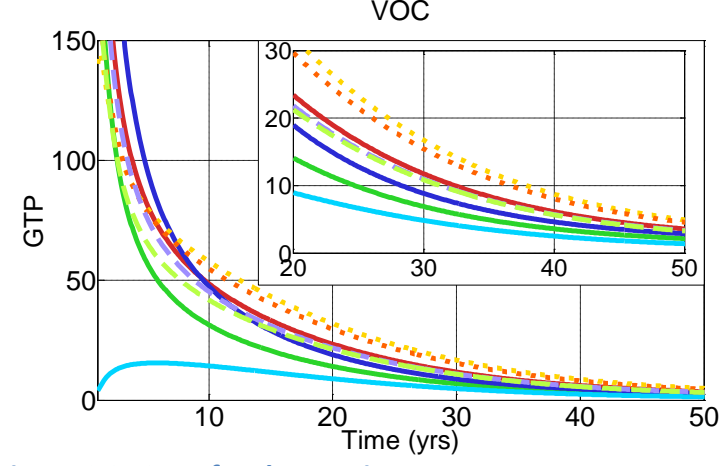

Figure SI2: GTPs for the species.

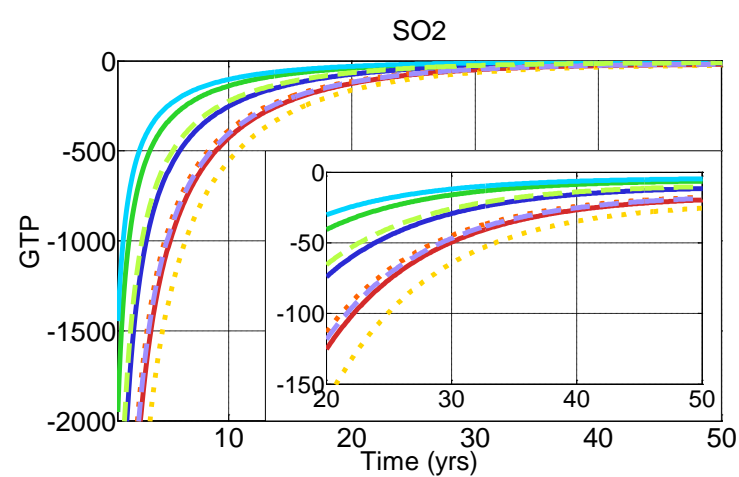

$\mathrm{CO}$

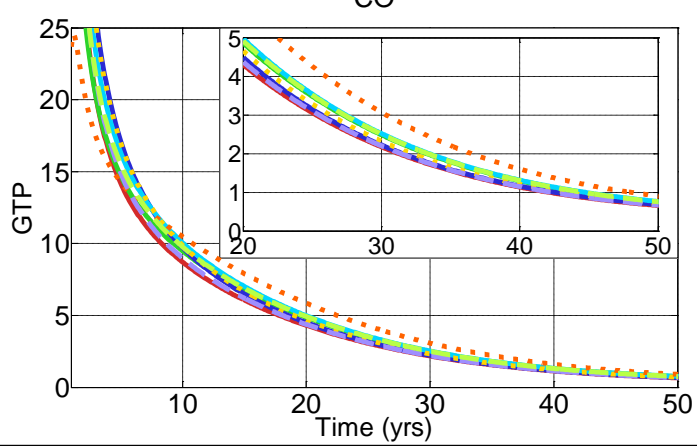

-Europe, summer

-Europe, winter

-East Asia, summer

-East Asia, winter

Shipping, NH summer

Shipping, NH winter

- Global, NH summer

Global, NH winter

42 
OC

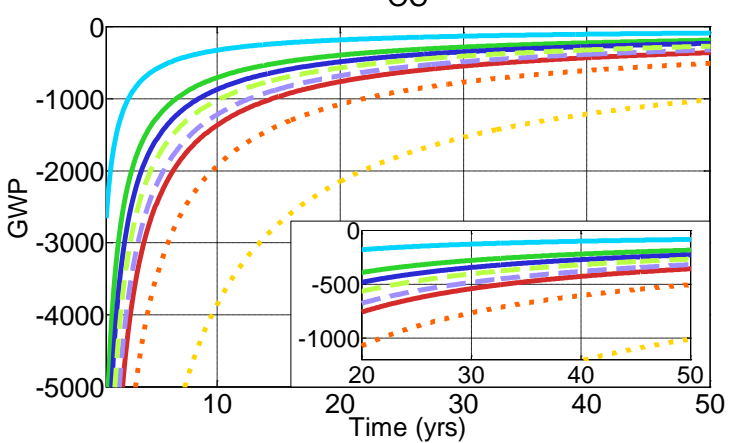

$\mathrm{NH} 3$

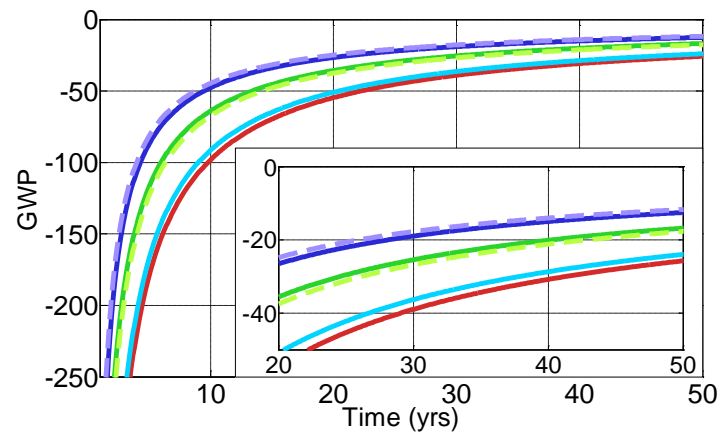

VOC

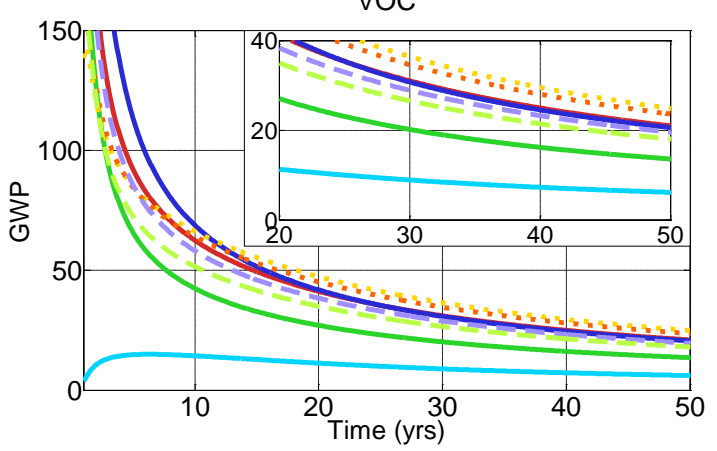

SO2

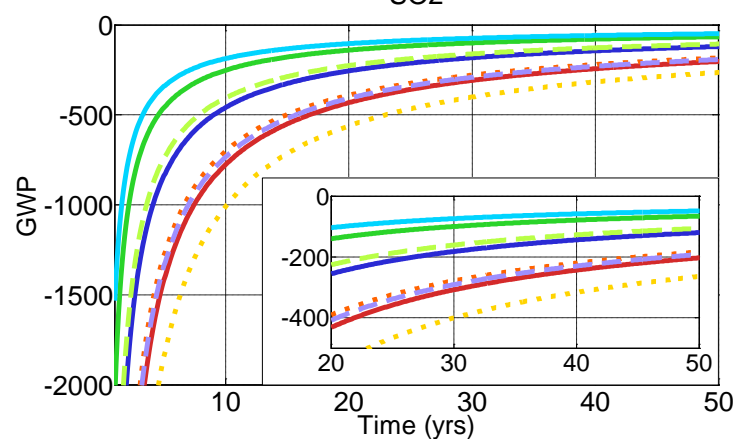

$\mathrm{CO}$

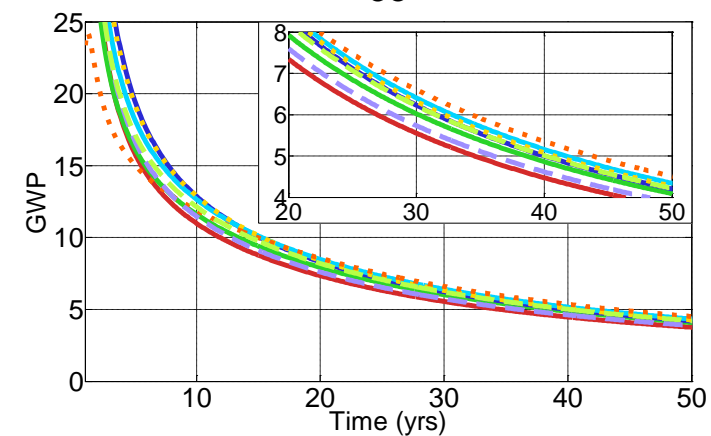

-Europe, summer

-Europe, winter

-East Asia, summer

-East Asia, winter

Shipping, NH summer

Shipping, $\mathrm{NH}$ winter

Global, NH summer

Global, NH winter

Figure SI3: GWPs for the species.

47

48

49

50

51

52

\section{Gradual implementation of mitigation}

Figure 9 presented how the emission metric values evolve with pulse emissions, sustained emissions, and a case of 15 years of ramp-up to illustrate a gradual implementation of mitigation policy. We present the same in Figure SI for the species not shown in the article. The ramp-up case is given for the different regions and seasons in Figure 10. Here, we include for those species not presented in the article, see Figure SI5 and Figure SI6. 


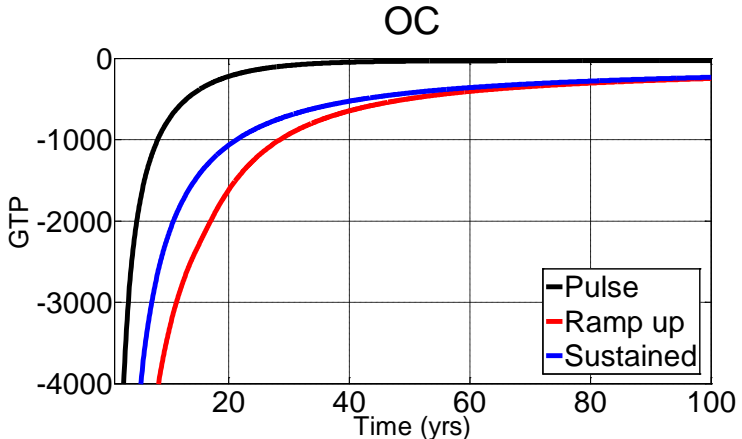

$\mathrm{SO} 2$

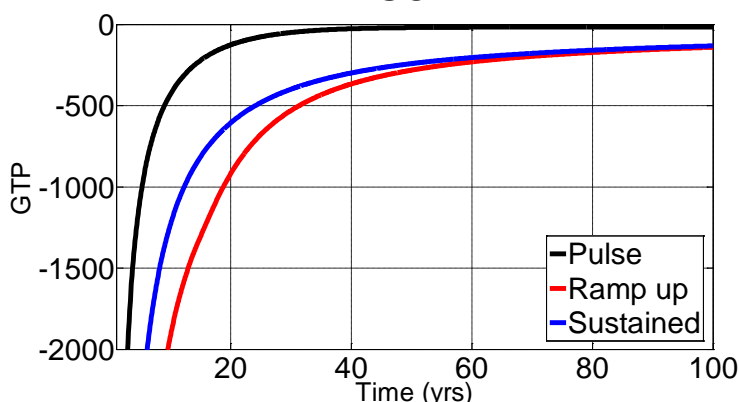

$\mathrm{NH} 3$

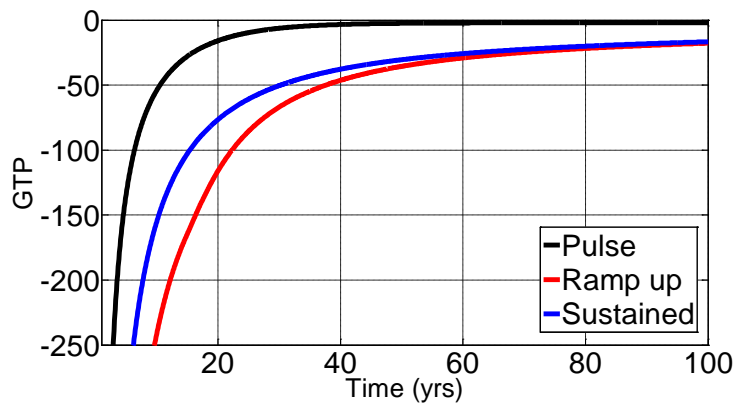

OC

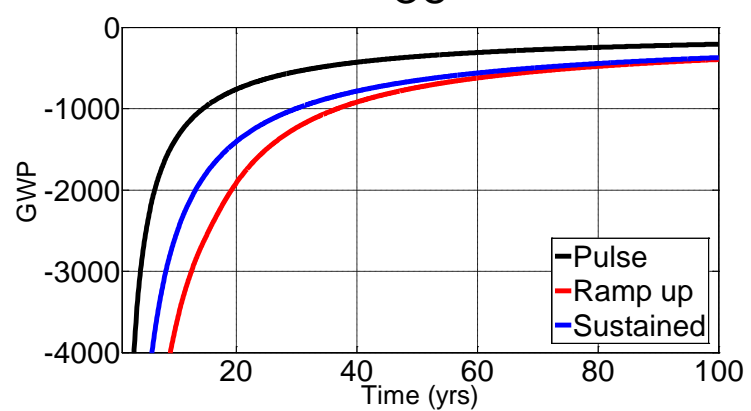

$\mathrm{SO} 2$

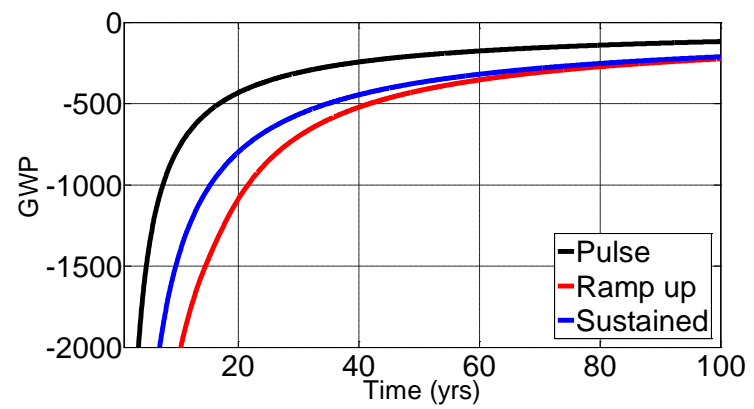

$\mathrm{NH} 3$

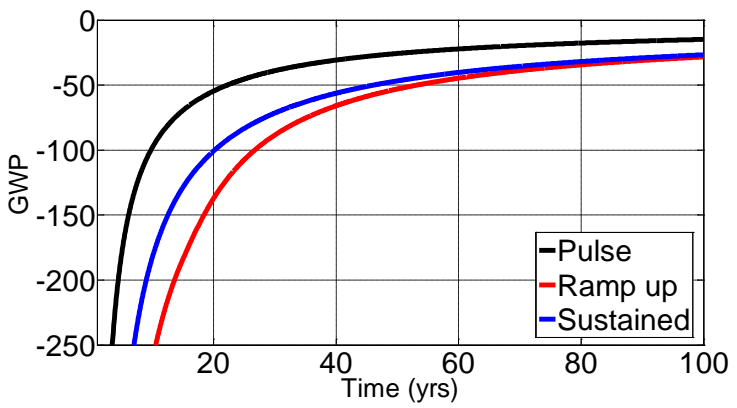

55

Figure SI4: The emission metric values for different types of emission profiles. GTP to the left and GWP to the right. 

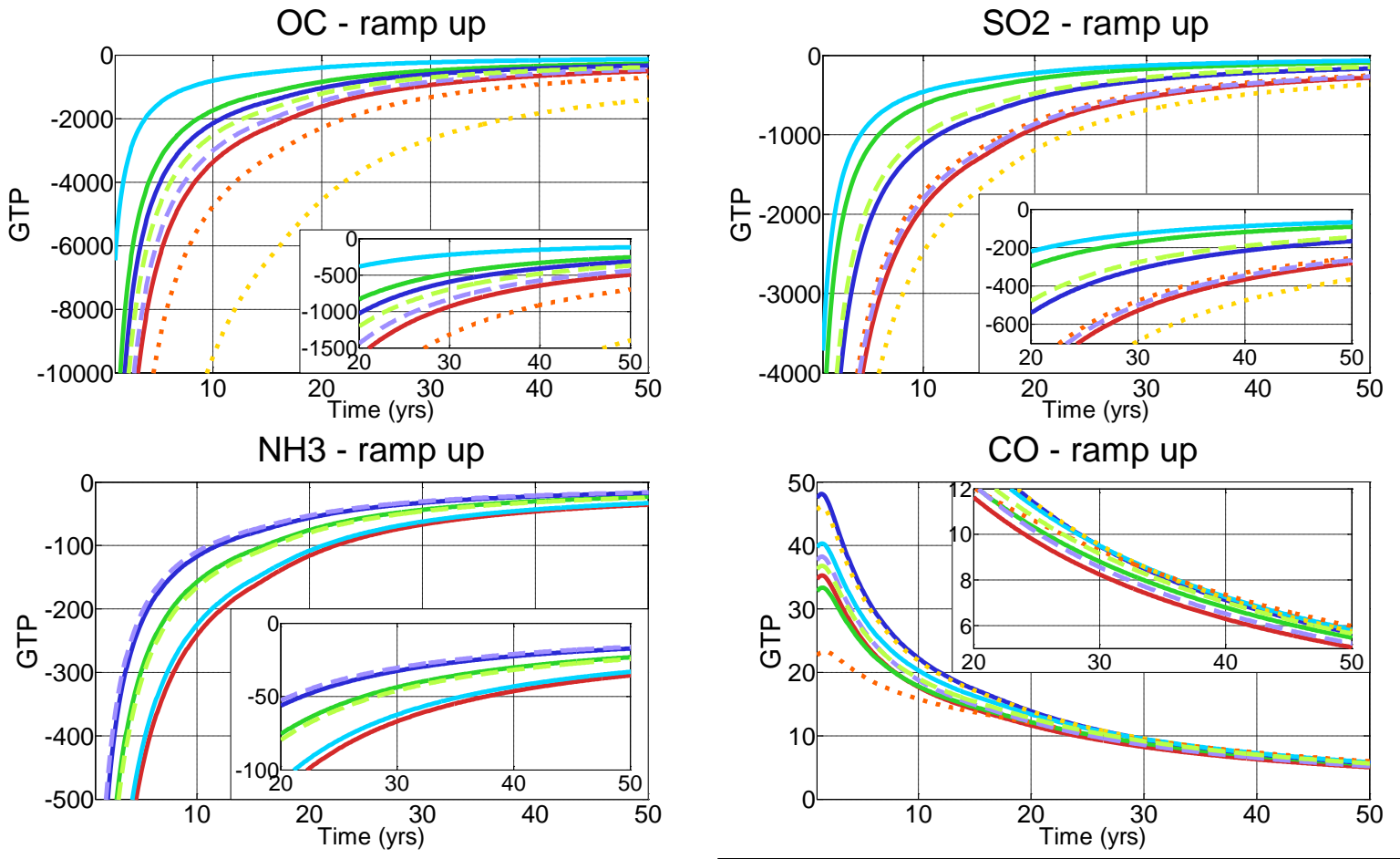

59

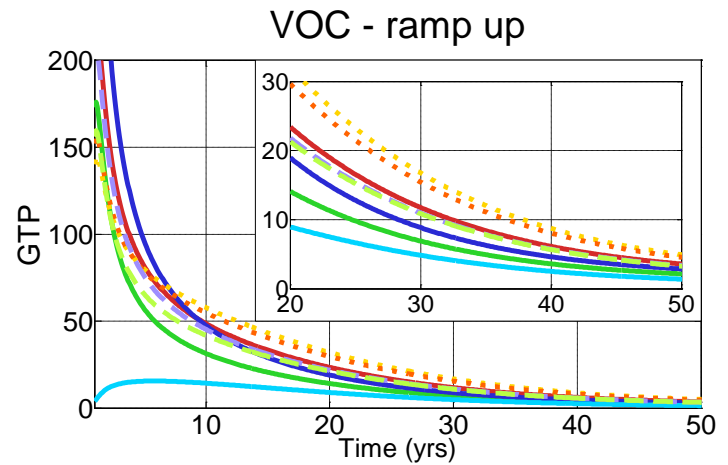

\begin{tabular}{l}
-Europe, summer \\
-Europe, winter \\
-East Asia, summer \\
-East Asia, winter \\
- Shipping, NH summer \\
- Shipping, NH winter \\
- Global, NH summer \\
- Global, NH winter \\
\hline
\end{tabular}

60

Figure SI5: GTPs for species with ramp-up emissions over 15 years. 


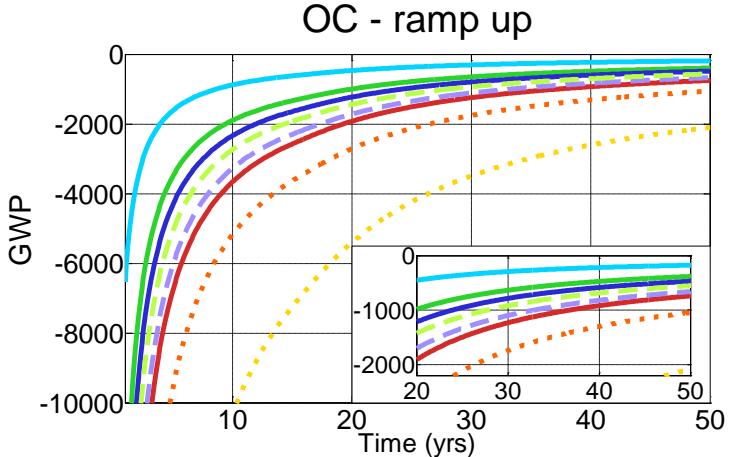

$\mathrm{NH} 3$ - ramp up

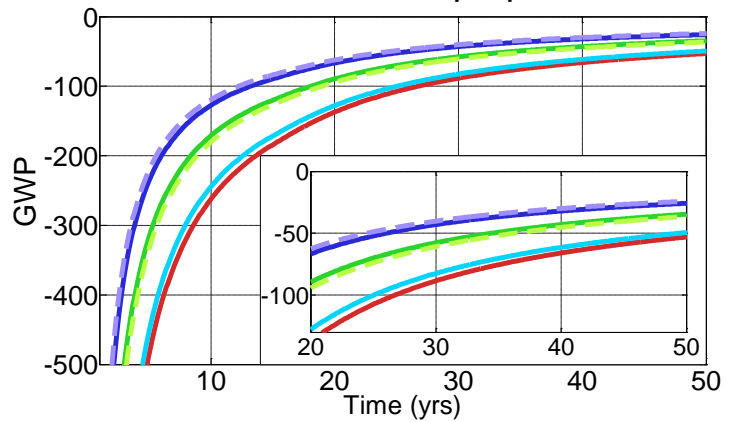

VOC - ramp up

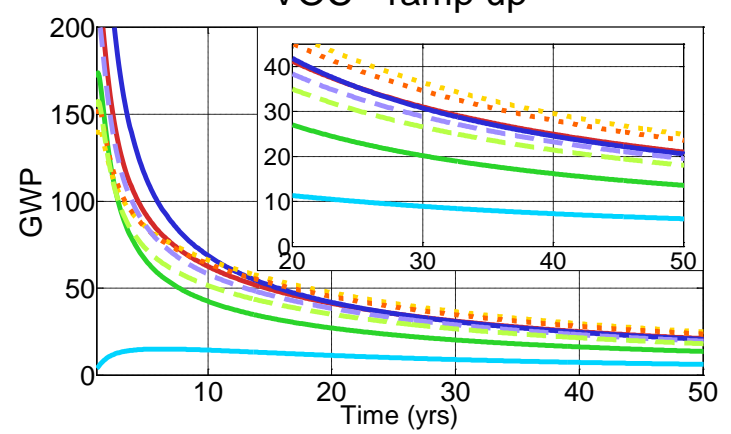

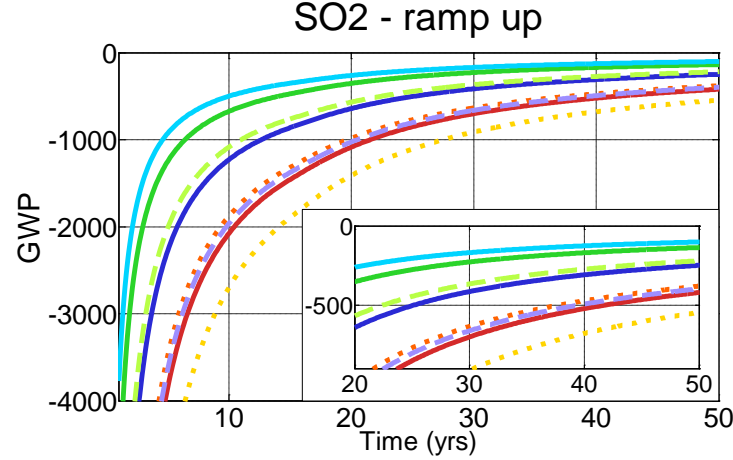

CO - ramp up

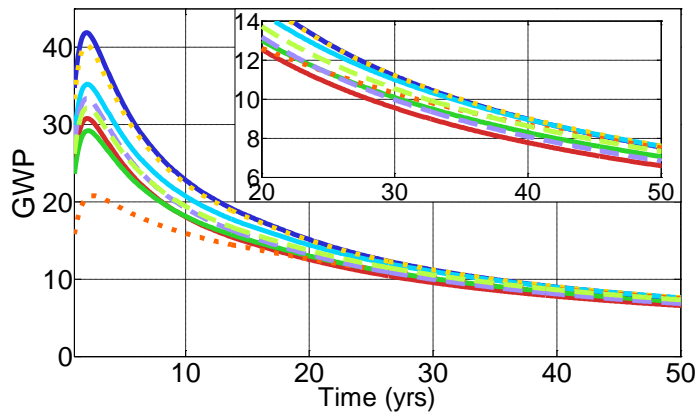

-Europe, summer

-Europe, winter

-East Asia, summer

-East Asia, winter

Shipping, NH summer

Shipping, NH winter

- Global, NH summer

Global, NH winter

Figure SI6: GWPs for species with ramp-up emissions over 15 years.

\section{References}

Aamaas, B., Peters, G., and Fuglestvedt, J. S.: Simple emission metrics for climate impacts, Earth Syst. Dynam., 4, 145-170, 10.5194/esd-4-145-2013, 2013.

Berntsen, T., Fuglestvedt, J. S., Joshi, M., Shine, K., Stuber, N., Li, L., Hauglustaine, D., and Ponater, M.: Climate response to regional emissions of ozone precursers: sensitivities and warming potentials, Tellus B, 57, 283-304, 2005.

Bond, T. C., Zarzycki, C., Flanner, M. G., and Koch, D. M.: Quantifying immediate radiative forcing by black carbon and organic matter with the Specific Forcing Pulse, Atmos. Chem. Phys., 11, 1505-1525, 10.5194/acp-11-1505-2011, 2011. Bond, T. C., Doherty, S. J., Fahey, D. W., Forster, P. M., Berntsen, T., DeAngelo, B. J., Flanner, M. G., Ghan, S., Kärcher, B., Koch, D., Kinne, S., Kondo, Y., Quinn, P. K., Sarofim, M. C., Schultz, M. G., Schulz, M., Venkataraman, C., Zhang, H., Zhang, S., Bellouin, N., Guttikunda, S. K., Hopke, P. K., Jacobson, M. Z., Kaiser, J. W., Klimont, Z., Lohmann, U., Schwarz, J. P., Shindell, D., Storelvmo, T., Warren, S. G., and Zender, C. S.: Bounding the role of black carbon in the climate system: A scientific assessment, Journal of Geophysical Research: Atmospheres, n/a-n/a, 10.1002/jgrd.50171, 2013.

Collins, W. J., Sitch, S., and Boucher, O.: How vegetation impacts affect climate metrics for ozone precursors, J. Geophys. Res., 115, D23308, 10.1029/2010jd014187, 2010.

Collins, W. J., Fry, M. M., Yu, H., Fuglestvedt, J. S., Shindell, D. T., and West, J. J.: Global and regional temperature-change potentials for near-term climate forcers, Atmos. Chem. Phys., 13, 2471-2485, 10.5194/acp-13-2471-2013, 2013.

Derwent, R. G., Collins, W. J., Johnson, C. E., and Stevenson, D. S.: Transient Behaviour of Tropospheric Ozone Precursors in a Global 3-D CTM and Their Indirect Greenhouse Effects, Climatic Change, 49, 463-487, 10.1023/a:1010648913655, 2001. Endresen, Ø., Sørgård, E., Sundet, J. K., Dalsøren, S. B., Isaksen, I. S. A., Berglen, T. F., and Gravir, G.: Emission from international sea transportation and environmental impact, Journal of Geophysical Research, 108, 4560, 2003.

Eyring, V., Stevenson, D. S., Lauer, A., Dentener, F. J., Butler, T., Collins, W. J., Ellingsen, K., Gauss, M., Hauglustaine, D. A., Isaksen, I. S. A., Lawrence, M. G., Richter, A., Rodriguez, J. M., Sanderson, M., Strahan, S. E., Sudo, K., Szopa, S., van Noije, T. 
P. C., and Wild, O.: Multi-model simulations of the impact of international shipping on Atmospheric Chemistry and Climate in 2000 and 2030, Atmos. Chem. Phys., 7, 757-780, 10.5194/acp-7-757-2007, 2007.

Fry, M. M., Naik, V., West, J. J., Schwarzkopf, D., Fiore, A., Collins, W. J., Dentener, F., Shindell, D. T., Atherton, C. S., Bergmann, D. J., Duncan, B. N., Hess, P. G., MacKenzie, I. A., Marmer, E., Schultz, M. G., Szopa, S., Wild, O., and Zeng, G.: The influence of ozone precursor emissions from four world regions on tropospheric composition and radiative climate forcing, J. Geophys. Res., 117, D07306, 10.1029/2011JD017134, 2012.

Fuglestvedt, J., Berntsen, T., Myhre, G., Rypdal, K., and Skeie, R. B.: Climate forcing from the transport sectors, Proceedings of the National Academy of Sciences, 105, 454-458, 10.1073/pnas.0702958104, 2008.

Fuglestvedt, J. S., Shine, K. P., Berntsen, T., Cook, J., Lee, D. S., Stenke, A., Skeie, R. B., Velders, G. J. M., and Waitz, I. A.: Transport impacts on atmosphere and climate: Metrics, Atmospheric Environment, 44, 4648-4677, 2010.

Köhler, M. O., Rädel, G., Dessens, O., Shine, K. P., Rogers, H. L., Wild, O., and Pyle, J. A.: Impact of perturbations to nitrogen oxide emissions from global aviation, Journal of Geophysical Research: Atmospheres, 113, D11305, 10.1029/2007JD009140, 2008.

Myhre, G., Shindell, D., Bréon, F.-M., Collins, B., Fuglestvedt, J. S., Huang, J., Koch, D., Lamarque, J.-F., Lee, D., Mendoza, B., Nakajima, T., Robock, A., Stephens, G., Takemura, T., and Zhang, H.: Anthropogenic and Natural Radiative Forcing, in: Climate Change 2013: The Physical Science Basis. Contribution of Working Group I to the Fifth Assessment Report of the Intergovernmental Panel on Climate Change, edited by: Stocker, T. F., Qin, D., Plattner, G. K., Tignor, M., Allen, S. K., Boschung, J., Nauels, A., Xia, Y., Bex, V., and Midgley, P. M., Cambridge University Press, Cambridge, United Kingdom and New York, NY, USA, 2013.

Naik, V., Mauzerall, D., Horowitz, L., Schwarzkopf, M. D., Ramaswamy, V., and Oppenheimer, M.: Net radiative forcing due to changes in regional emissions of tropospheric ozone precursors, J. Geophys. Res., 110, D24306, 10.1029/2005jd005908, 2005.

Shindell, D. T., Faluvegi, G., Koch, D. M., Schmidt, G. A., Unger, N., and Bauer, S. E.: Improved Attribution of Climate Forcing to Emissions, Science, 326, 716-718, 10.1126/science.1174760, 2009.

Stevenson, D. S., Doherty, R. M., Sanderson, M. G., Collins, W. J., Johnson, C. E., and Derwent, R. G.: Radiative forcing from aircraft NOx emissions: Mechanisms and seasonal dependence, J. Geophys. Res., 109, D17307, 10.1029/2004jd004759, 2004.

Wild, O., Prather, M. J., and Akimoto, H.: Indirect long-term global radiative cooling from NOx emissions, Geophys. Res. Lett., 28, 1719-1722, 10.1029/2000gl012573, 2001. 